Original

\title{
Nuevas dianas terapéuticas para el carcinoma de células renales metastásico. Resultados del tratamiento con bevacizumab en monoterapia en un único centro hospitalario
}

Verónica Calderero Aragón*, Roberto Pazo Cid, Teresa Puértolas Hernández*, Angel Artal Cortés*, Angel Borque Fernando**, $\mathrm{M}^{\mathrm{a}}$ Jesús Gil Sanz**

*Servicio de Oncología Médica. **Servicio de Urología. Hospital Universitario Miguel Servet. Zaragoza, España

\section{Resumen}

Objetivos: En la última década se están desarrollando multitud de nuevas moléculas que ofrecen resultados esperanzadores para el tratamiento del CCR metastásico. Este estudio describe nuestra experiencia en 25 pacientes tratados con bevacizumab en monoterapia.

Material y métodos: Se analizan los resultados obtenidos en 25 pacientes con CCR metastásico tratados con Bevacizumab (10 mg/Kg quincenal) entre enero del 2006 y enero del 2008. Se recogieron datos acerca del tipo de cirugía practicada, tipo histológico, estadio tumoral, grupo de riesgo de Motzer, localizaciones metastásicas, número de ciclos administrados, tiempo hasta la progresión y supervivencia global.

Resultados: Se analizaron 25 pacientes (16 varones y 9 mujeres), con una mediana de edad de 59 años. Más de la mitad de ellos (19) se había sometido a algún tipo de intervención quirúrgica (14 nefrectomía radical, 4 citorreductora y 1 tumorectomía). La mayor parte de los enfermos presentaban histología de células claras (22). 14 pacientes pertenecían al grupo de buen pronóstico de Motzer, 8 al grupo de pronóstico intermedio y 3 al de mal pronóstico.

La mediana de tiempo hasta la progresión fue de 19 semanas, con una mediana de supervivencia global desde el inicio de tratamiento de 8,7 meses.

Conclusiones: El bevacizumab en monoterapia ofrece unos resultados modestos para el tratamiento del CCR metastásico. Su reciente aprobación en combinación con Interferon, y la aparición progresiva de distintas dianas terapéuticas, permitirá optimizar los resultados en los próximos años.

Palabras clave: Bevacizumab. Carcinoma renal. Nuevos agentes.

\section{Novel therapeutic targets in metastatic renal cell carcinoma. Results of the treatment with single-agent bevacizumab in a single institution}

\section{Abstract}

Aim: Along the past decade a number of new agents have been developed showing promising results in the treatment of metastatic renal cell carcinoma. We describe our experience with the use of bevacizumab in monotherapy in the Urology and Medical Oncology departments of our institution.

Material and methods: A consecutive series of patients treated with Bevacizumab $10 \mathrm{mg} / \mathrm{Kg}$ every 14 days between January 2006 and January 2008 has been assessed. Data concerning the type of surgery, histological subtype, tumor stage, Motzer's risk group, metastatic sites number of bevacizumab courses given, time to progression and overall survival were collected.

Results: 25 patients (16 male, 9 female) were analyzed. Median age was 59 years. Nineteen of them (75\%) had prior surgery: radical nephrectomy 14, cytoreductive 4 y tumorectomy 1). Clear cell carcinoma was the most frequent subtype (22 patients). According to Motzer's prognostic index 14 had good prognosis, 8 intermediate and 3 poor prognosis.

Median time to progression was 19 weeks. Overall median survival since metastatic disease was diagnosed 8,7 months. Conclusions: Bevacizumab offers, as single agent, modest activity in the metastatic renal cell carcinoma. The recently labeled use in combination with interferon as well as the newer targeted agents will improve the results of the treatment of this disease in the next future.

Keywords: Bevacizumab, Renal cell carcinoma. Novel agents.

$\mathrm{E}^{1}$ carcinoma de células renales (CCR) es un tumor de gran agresividad y de conocido mal pronóstico debido a la limitación de las alternativas terapéuticas presente hasta hace pocos años.
La resección quirúrgica es el pilar del tratamiento para aquellos tumores confinados al riñón. Sin embargo, hasta el 30\% de los casos resecados llegarán a desarrollar metástasis a distancia a lo largo de 
su evolución. La inmensa mayoría de estos pacientes no podrán curarse y los tratamientos con inmunoterapia, interferon (IFN) o interleukina-2 (IL-2), obtienen medianas de supervivencia de 12 a 17 meses $^{1-5}$.

En las últimas décadas el CCR se ha convertido en el modelo ideal para el desarrollo de agentes dirigidos a dianas moleculares: el carcinoma de células claras constituye el subtipo histológico más frecuente y se caracteriza por la inactivación del gen de Von Hippel-Lindau (VHL). La inactivación de este gen supresor tumoral altera la regulación de genes asociados a la hipoxia y desencadena una cascada de sobreproducción de moléculas promotoras de la angiogénesis, la proliferación y la diseminación tumoral.

Una vez descubierto que la vía del gen VHL y el factor de crecimiento endotelial vascular (VEGF) es crítica para el desarrollo del cáncer renal ha habido diferentes agentes como bevacizumab, sorafenib, sunitinib o temsirolimus que han conseguido su aprobación para el tratamiento de este tumor. Además, a día de hoy existen numerosas moléculas en fase de desarrollo.

El bevacizumab (Bv) es un anticuerpo monoclonal recombinante que se une y neutraliza isoformas biológicas del $\mathrm{VEGF}^{6}$. Fue una de las primeras moléculas en mostrar actividad contra el cáncer renal tanto en estudios preclínicos como en distintos estudios Fase $\mathrm{I}^{7-9}$. Este fármaco ha demostrado un significativo beneficio clínico en distintos estudios Fase II de CCR en estadio IV ${ }^{10-12}$.

En monoterapia obtiene medianas de tiempo a la progresión en torno a 8,5 meses en pacientes no tratados previamente. En pacientes ya tratados previamente consigue aumentar la mediana de tiempo hasta la progresión comparado con placebo $(4,8$ meses frente a 2,5 meses, $\operatorname{HR} 0,39, \mathrm{p}<0,001)$. Existe ,además, un pequeño porcentaje de pacientes que obtienen respuestas duraderas que superan los 3-5 años.

Tras observar que el tratamiento con Bv retrasaba la progresión del tumor se intentó combinar el fármaco con agentes tumoricidas para mejorar los resultados. De esta forma, a finales de 2007 se publicaron en Lancet los resultados del estudio Fase III en primera línea de tratamiento de IFN a con $\mathrm{Bv}$ frente a IFN en monoterapia ${ }^{13}$. Este estudio condujo a la aprobación del fármaco por la FDA, al demostrar beneficio de la combinación IFN $+\mathrm{Bv}$ frente a
IFN sólo, tanto en términos de tasa de respuestas como de tiempo hasta la progresión.

En este artículo se describen los resultados obtenidos en nuestro centro en pacientes tratados con $\mathrm{Bv}$ en monoterapia, antes de la aprobación en nuestro país de otros fármacos que se utilizan en la actualidad.

\section{MATERIAL Y MÉTODOS}

Se analizaron retrospectivamente las historias clínicas de todos los pacientes tratados con $\mathrm{Bv}$ en monoterapia entre enero de 2006 y enero de 2008.

De la documentación clínica se extrajeron los siguientes datos: sexo, edad, tipo de cirugía practicada, subtipo histológico, estadio tumoral al diagnóstico, número y localizaciones metastásicas, criterios de riesgo de Motzer, administración previa o no de tratamiento con inmunoterapia, número de ciclos de $\mathrm{Bv}$ administrados, respuesta objetiva, efectos secundarios, tiempo hasta la progresión y supervivencia.

Se realizó una descripción estadística de las caracteríticas de los pacientes y del tratamiento administrado. El tiempo hasta la progresión y la supervivencia global se calcularon a partir del momento de inicio de tratamiento, según el método Kaplan-Meier.

El análisis estadístico se realizó usando la versión SPSS Inc 15.0 Statistical Software.

\section{RESULTADOS}

Se analizaron las historias clínicas de 25 pacientes tratados durante el período descrito, cuyas características clínicas y demográficas se describen en las Tablas 1 y 2 .

La media de edad de los pacientes fue de 55 años (40-78). La mayor parte de ellos (76\%) habían sufrido algún tipo de cirugía sobre el tumor, bien con intención radical o citorreductora, ya en el contexto de enfermedad avanzada. La histología más frecuente $(88 \%)$ fue de carcinoma de células claras, mientras que el resto incluía otras variedades (sarcomatoide y papilar).

Sólo el 44\% de los pacientes remitidos para tratamiento eran metastásicos en el momento del diagnóstico. $\mathrm{El} 28 \%$ de los pacientes desarrollaron metástasis más allá de 12 meses tras el diagnóstico inicial, y el resto lo hicieron en los primeros 12 meses. Las localizaciones metastásicas más frecuentes fueron; pulmón (24\%), hueso (8\%), hígado (4\%), ganglionar (4\%) o múltiples (60\%). 
Tabla 1. Características demográficas de los 25 pacientes

\begin{tabular}{|c|c|}
\hline & $\mathbf{N}^{\mathbf{o}}$ \\
\hline \multicolumn{2}{|l|}{ Sexo } \\
\hline Varones & 16 \\
\hline Mujeres & 9 \\
\hline \multicolumn{2}{|l|}{ Cirugia } \\
\hline Nefrectomía radical & 14 \\
\hline Nefrectomía citorreductora & 4 \\
\hline Tumorectomía & 1 \\
\hline Ninguna & 6 \\
\hline \multicolumn{2}{|l|}{ Histologia } \\
\hline Células claras & 22 \\
\hline Otros & 3 \\
\hline \multicolumn{2}{|l|}{ Estadio inicial } \\
\hline I & 2 \\
\hline II & 6 \\
\hline III & 6 \\
\hline IV & 11 \\
\hline \multicolumn{2}{|l|}{ Grupo riesgo de Motzer } \\
\hline Buen pronóstico & 14 \\
\hline Pronóstico intermedio & 8 \\
\hline Mal pronóstico & 3 \\
\hline
\end{tabular}

Tabla 2. Características clínicas de los 25 pacientes

\begin{tabular}{lc}
\hline & $\mathbf{N}^{\mathbf{0}}$ \\
\hline $\begin{array}{c}\text { Aparición de M1 tras diagnóstico } \\
<12 \text { meses }\end{array}$ & 18 \\
$>12$ meses & 7 \\
Localizaciones metastásicas & \\
Pulmón & 6 \\
Hueso & 2 \\
Hígado & 1 \\
Ganglionar & 1 \\
Múltiples & 15 \\
Momento inicio tratamiento & \\
Como primera línea & 18 \\
Tras inmunoterapia & 7 \\
No de ciclos & \\
Mediana & $(2-54)$ \\
Respuestas & \\
RC & 0 \\
RP & 6 \\
EE & 11 \\
PE & 8 \\
Toxicidad G2-3 & \\
HTA & 9 \\
Astenia & 1 \\
Otras & 3 \\
Ninguna & 12 \\
\hline
\end{tabular}

Algo más de la mitad de los enfermos cumplían criterios que los incluían en el grupo de buen pronóstico de Motzer (56\%); el 32\% pertenecían al grupo de pronóstico intermedio y el $12 \%$ eran de mal pronóstico.
En el $72 \%$ de los casos se inició el tratamiento con Bv como primera línea de tratamiento de enfermedad metastásica, mientras que el $28 \%$ lo hicieron tras inmunoterapia.

Se llegó a administrar una mediana de 8 ciclos por paciente, aunque el intervalo fue desde 2 hasta 54 ciclos. El $16 \%$ de los casos recibieron tratamiento continuado más allá de 12 meses, sin presentar ningún tipo de complicación asociada al fármaco.

No se alcanzó ninguna respuesta completa, en el $24 \%$ de los pacientes se objetivó respuesta parcial. Un 44\% de ellos obtuvieron estabilización como mejor respuesta. Un $32 \%$ de los pacientes presentaron progresión de sus enfermedad a pesar del tratamiento. Por tanto, la tasa de beneficio clínico se sitúa en el $68 \%$.

La tolerancia al fármaco fue excelente. La toxicidad grado 2-3 más relevante fue la hipertensión arterial (HTA) que apareció en un 36\% de pacientes. Con antihipertensivos habituales se consiguió un adecuado control de la misma en todos los casos. No se produjo ninguna hemorragia, tampoco hubo de interrumpirse la administración del fármaco por toxicidad, ni hubo ninguna muerte tóxica.

Otras toxicidades menos frecuentes fueron la astenia (4\%) y la proteinuria $(12 \%)$. El resto de molestias asociadas al tratamiento (anorexia, cefalea, etc) fueron de carácter leve (grado 1).

En el momento del análisis se habían producido 16 muertes, todas ellas asociadas al tumor o sus complicaciones. Se obtuvo una mediana de tiempo a la progresión de 19 semanas (IC 95\% 11,24-26,75), con una supervivencia global tras el inicio de tratamiento 8,7 meses (IC 95\% 4,55-12,84) Figs. 1 y 2.

\section{DISCUSIÓN}

La actividad de $\mathrm{Bv}$ en monoterapia fue valorada por primera vez por Yang y colaboradores ${ }^{10-11}$ en un ensayo controlado con placebo en pacientes con carcinoma de células renales metastásico y refractario a citocinas. Los datos preclínicos y también clínicos procedentes de un estudio Fase II de un único brazo con Bv más erlotinib demostraban su activi$\operatorname{dad}^{14-15}$.

Nuestra serie de casos corresponde a un número recortado de pacientes que se trataron con el fármaco en monoterapia, de manera previa a la aprobación reciente de nuevas dianas y a la publicación del estudio Fase III de combinación con IFN alfa. 


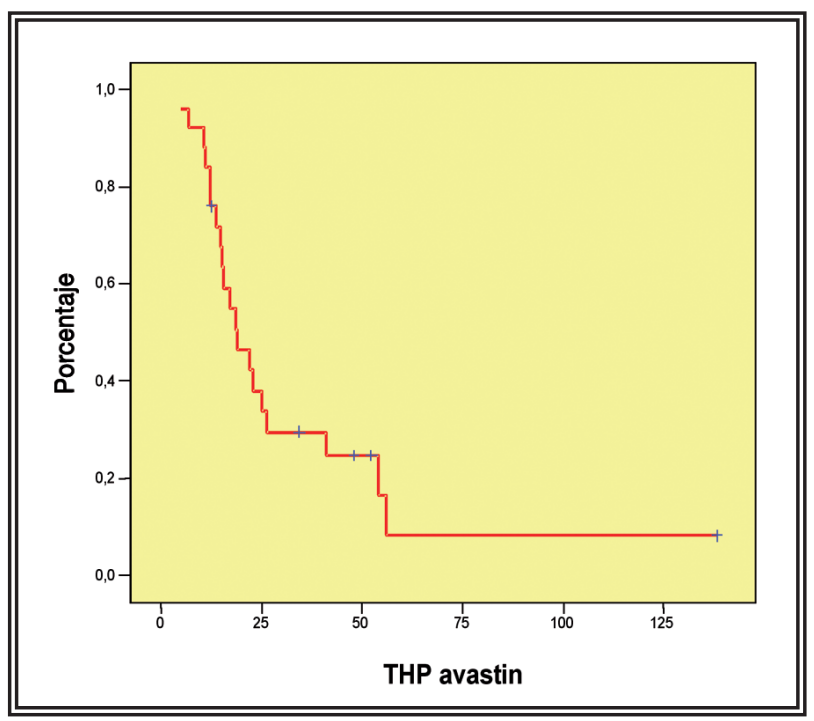

FIGURA 1. Gráfica de Kaplan-Meier que muestra la evolución del tiempo hasta la progresión de los pacientes de la serie.

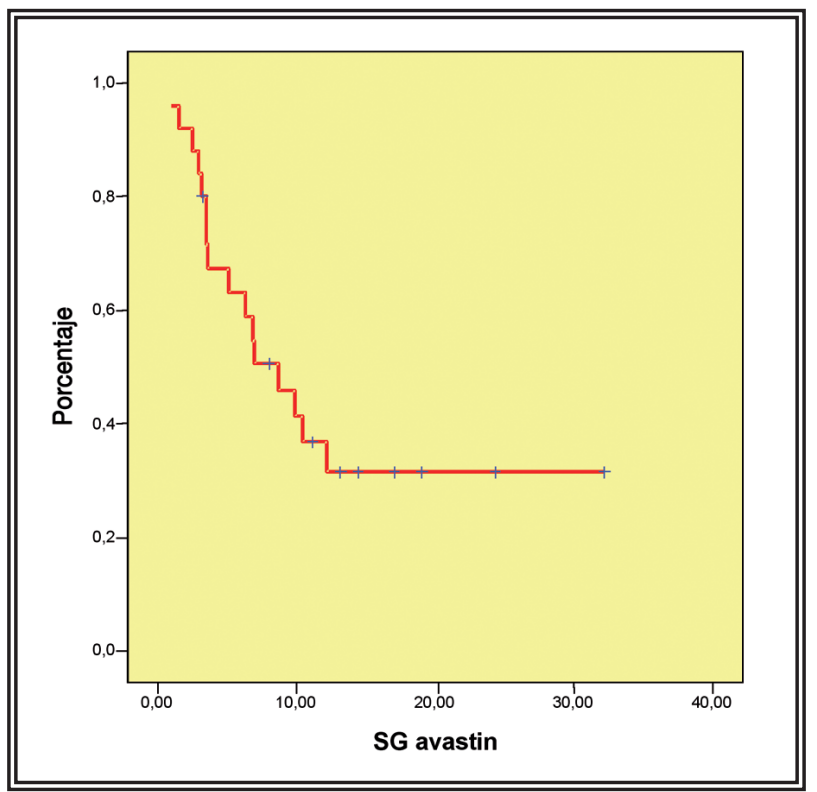

FIGURA 2. Gráfica de la supervivencia global de los pacientes.

Comparar nuestros resultados con los publicados previamente es complicado. En primer lugar la serie es pequeña y el seguimiento corto. Además la población es heterogéna y no comparable con las de estudios previos: incluímos pacientes en primera línea de tratamiento y en progresión a citocinas y de los tres grupos de riesgo de Motzer.

Lo que parece evidente es que este fármaco en monoterapia tiene una actividad limitada y con los datos disponibles a día de hoy, debería intentarse su uso en combinación con otros agentes.
La tolerancia al bevazucimab es excelente. La HTA es el efecto secundario más frecuente, pero suele controlarse adecuadamente con terapia antiHTA. La proteinuria no suele tener repercusión en la función renal, aunque debe controlarse. Y la trombosis y perforación gastrointestinal, presentes en pacientes con carcinoma colorrectal tratados, son extraordinariamente infrecuente en los casos de cáncer renal.

No obstante, en los últimos meses se han publicado distintos estudios Fase III ${ }^{16-18}$ que han permitido que tengamos disponibles de forma asistencial nuevas dianas terapéuticas como el sunitinib, sorafenib y temsirolimus.

El uso de estos medicamentos debe de seleccionarse en función de las características del paciente, del tipo de tumor, de la vía de administración del fármaco y de su toxicidad, así como de su farmacoeconomía ${ }^{19-21}$.

La multitud de ensayos clínicos abiertos y de moléculas en desarrollo, probablemente provoque el que el panorama de tratamiento del cáncer renal cambie radicalmente en los próximos años. En un tumor considerado resistente a los tratamientos hasta hace pocos años, ¿quizás el futuro nos ofrezca la oportunidad de hablar de tasas de curación?..

\section{REFERENCIAS}

1. Negrier S, Escudier B, Lasset C, Douillard JY, Savary J, Chevreau C, et al. Recombinant human interleukin-2, recombinant human interferon alpha-2a, or both in metastatic renal-cell carcinoma. N Engl J Med. 1998;338(118):1272-1278.

2. Fyfe G, Fisher RI, Rosenberg SA, Sznol M, Parkinson DR, Louie AC. Results of treatment of 255 patients with metastatic renal cell carcinoma who received high-dose recombinant interleukin2 therapy. J Clin Oncol. 1995;13(3):688-96.

3. McDermott DF, Regan MM, Clark JI, et al. Randomized phase III trial of high dose interleukin-2 versus subcutaneous intrleukin2 and interferon in patients with metastatic renal cell carcinoma. J Clin Oncol 2005; 23(1):133-41.

4. Motzer RJ, Murphy BA, Bacik J, et al. Phase III trial of interferon alfa-2a with or without 13-cis-retinoic acid for patients with advanced renal cell carcinoma. J Clin Oncol 2000; 18(6)297280 .

5. Aass N, De Mulder HM, Mickisch GHJ, et al. Randomized phase II/III trial of interferon alfa-2a with or without 13-cisretinoic acid in patients with progressive metastasic renal cell carcinoma: the European Organization for Research and Treatment of Cancer Genito-Urinary Tract Cancer Group (EORTC 30951). J Clin Oncol 2005;23(18):4172-178.

6. Presta LG, Chen H, O'Connor SJ, et al. Humanization of an antiVEGF monoclonal antibody for the therapy of solid tumors and other disorders. Cancer Res 1997;57(20):4593-4599.

7. Dagnaes-Hansen F, Rasmussen LM, Tilton R, Denner L, Flyvbjerg A. A murine VEGF antibody inhibits in vivo growth of human Caki-I renal adenocarcinoma. Anticancer Res 2003; 232B): 1625-1630 
8. Margolin K, Gord on MS, Holmgren E, et al. Phase Ib trial of intravenous recombinant humanized antibody to VEGF in combination with chemotherapy in patients with advanced cancer: pharmacologic and long-term safety data. J Clin Oncol 2001;19:851-856.

9. Gordon MS, Margolin K, Talpaz M, et al. Phase I safety and pharmacokinetic study of recombinant human anti-VEGF in patients with advanced cancer. J Clin Oncol 2001;19(3):843850.

10. Yang JC, Haworth L, Sherry RM, et al. A randomized trial of Bv, an anti-VEGF antibody, for metastatic renal cancer. N Engl J Med 2003;349(5):427-434.

11. Yang JC: Bv for patients with metastatic renal cancer: an update. Clin cancer Res 2004; 15;10(18 Pt 2):6367S-6370S.

12. Escudier B, Koralewski P, Pluzanska A, et al. A randomized, controlled, double blind phase III study (AVOREN) of Bv/IFNa2a vs placebo/ IFNa-2a as first line therapy in metastatic RCC. Prom Am Soc Clin Oncol 2007;25:18S.

13. Escudier B, Pluzanska A, Koralewski P, Ravaud A, Bracarda S, Szcylik C, Chevreau C, Filipak M, Melichar B, Bajetta E, Gorbunova V, Bay JO, Bodrogi I, Jagiello-Gruszfeld A, Moore N. Bv plus interferon alfa-2a for treatment of metastatic renal cell carcinoma: a randomized, double blind fase III trial. Lancet 2007;370(9605):2103-2111.

14. Hainsworth JD, Sosman JA, Spigel DR et al. Treatment of metastatic renal cell carcinoma with combination of $\mathrm{Bv}$ and erlotinib. J Clin Oncol 2005;23(31):7889-7896.
15. Bukowski RM, Kabbinavar F, Figlin RA, et al. Bv with or without erlotinib in metastatic renal cell acrcinoma. J Clin Oncol 2006;24:222S.

16. Motzer RJ, Hutson TE, Tomczak P, et al. Sunitinib versus IFNa in metastatic RCC. N Engl J Med 2007;356(2):115-124.

17. Escudier B, Eisen T, Stadler WM, et al. Sorafenib in advanced clear -cell carcinoma. N Engl J Med 2007;356(2):125-134.

18. Hudes G, Carducci M,Tomczak P, et al. Temsirolimus, IFNa or both for advanced RCC. N Engl J Med 2007;356(22):2271-2281.

19. Motzer RJ, Hoosen S, Bello CL, Christensen JG. Sunitinib malate for the treatment of solid tumours: a review of current clinical data. Expert Opin Drugs(2006) 15(5):553-561.

20. Frampton JE, Keating GM. Bv in first line treatment of advanced or metastatic renal cell carcinoma. Biodrugs 2008; 22(2):113-20.

21. García JA, Rini BI. Recent progress in the management of advanced renal cell carcinoma. CA Cancer J Clin 2007 Mar; 57 (2):112-125.

Correspondencia autor: Dra. Verónica Calderero Aragón Servicio de Oncología Médica. Hospital Universitario Miguel Servet $\mathrm{P}^{\circ}$ Isabel La Católica, 1-3 - 50009 Zaragoza

Tel.: 976765500

E-mail autor: dracalde@hotmail.com

Información artículo: Original - Cáncer renal

Trabajo recibido: septiembre 2008

Trabajo aceptado: noviembre 2008 